\title{
SOFTWARES PARA OS SERVIÇOS DE SAÚDE: UMA REVISÃO INTEGRATIVA A RESPEITO DE PESQUISAS BRASILEIRAS
}

\begin{abstract}
Iramara Lima Ribeiro
Doutoranda e Mestra em Saúde Coletiva pela Universidade Federal do Rio Grande do Norte (UFRN), com Especialização em Vigilância Sanitária e graduação em Nutrição, possui experiência na área de Saúde Coletiva, com ênfase em Educação, Formação e Processo de Trabalho em Saúde. E-mail: iramararibeiro@ hotmail.com

Iris do Céu Clara Costa

Pós-doutora em Psicologia Social pela Universidade Aberta de Lisboa, doutora em Odontologia Preventiva e Social, Mestre em Odontologia Social, é Professora Associada

III da UFRN, atuando principalmente nos temas de Saúde Ocupacional, Processo de Trabalho e Educação em Saúde. E-mail: iris_odontoufrn@ yahoo.com.br

José Guilherme da Silva Santa Rosa

Doutor em Educação em Ciências e Saúde pela Universidade Federal do Rio de Janeiro, com mestrado em Design, especialização em Computação Gráfica e Multimídia e graduação em Análise e Desenvolvimento de Sistemas, é Professor Adjunto II da UFRN, possui experiências nas áreas de Ergonomia Funcional, Usabilidade, Interação Humano

Computador e Programação Visual. E-mail: jguilhermesantarosa@gmail.com
\end{abstract}

\section{RESUMO}

A população brasileira vem enfrentando modificações nos padrões de morbimortalidade, podendo as tecnologias auxiliar na organização dos serviços e prestação do cuidado. Esta revisão integrativa teve por objetivo analisar as contribuições do desenvolvimento de softwares no Brasil para a organização e a integração das ações e dos serviços de saúde. Foram analisados 17 materiais entre 2008 e 2013, sendo a maioria dos autores do Sudeste brasileiro, sinalizando uma quase hegemonia desta região. Quanto à finalidade principal, $47 \%$ destinava-se a organização dos serviços, 29,4\% ao diagnóstico e 23,6\% ao monitoramento, prevenção e tratamento de doenças. Todos eles possuíam relação com a vigilância em saúde e nenhum abordava a promoção da saúde. Os usuários finais eram em maior parte profissionais da saúde $(70,6 \%)$. Apesar de positiva a presença de softwares para organização dos serviços e diagnóstico, a carência de estudos quanto ao monitoramento, tratamento e prevenção de doenças, com ausência daqueles para a promoção da saúde, evidencia uma valorização de estudos voltados para a doença quando se poderia trabalhar também no sentido de evitar causas preveníveis. As proposições dos softwares podem trazer benefícios, mas aponta-se a necessidade de ampliar as opções de uso quanto à finalidade e aos usuários.

PALAVRAS-CHAVE: Saúde, Software, Serviços de Saúde, Organização dos Serviços. 


\title{
SOFTWARE FOR HEALTH SERVICES: NA INTEGRATIVE REVIEW ABOUT BRAZILIAN RESEARCH
}

\begin{abstract}
The Brazilian population is facing changes in patterns of morbidity and mortality, and technologies may assist in the organization of services and healthcare. The aim of this integrative review was to analyze the contributions of software development in Brazil for the organization and integration of actions and health services. Seventeen materials were analyzed between 2008 and 2013, with most authors of Southeastern Brazil, signaling an almost hegemony of this region. Concerning the main purpose, $47 \%$ intended to organization of services, $29.4 \%$ to diagnosis and $23.6 \%$ to monitoring, prevention and treatment of diseases. They all possessed relation with health surveillance and neither of them with health promotion. The mainly end users were health professionals $(70.6 \%)$. Despite the positive presence of software for organization of services and diagnosis, the need for studies on the monitoring, treatment and prevention of diseases, with the absence of those for health promotion, shows a valorization of studies on the disease when it could work also in order to avoid preventable causes. The propositions of software can bring benefits, but there are a need to expand the usage options as to the purpose and users.
\end{abstract}

KEY WORDS: Health, Software, Health Services, Organization of Services. 


\section{SOFTWARES PARA OS SERVIÇOS DE SAÚDE: UMA REVISÃO INTEGRATIVA A RESPEITO DE PESQUISAS BRASILEIRAS}

\section{INTRODUÇÃO}

Os padrões de morbimortalidade vêm se modificando na população brasileira, estando como principais causas de óbito e internamentos, as doenças crônico-degenerativas, os acidentes e as diversas formas de violência, além de doenças como a cólera e o dengue ressurgidas recentemente e a persistência de problemas como a desnutrição, cujo tratamento envolve recursos tecnológicos, onerando o sistema de saúde (BARRETO E CARMO, 2007).

Nesta perspectiva, o uso de novas tecnologias tem ganhado espaço na área da saúde, estendendo-se desde a organização dos serviços até a prestação do cuidado e devendo para tal, estar atrelado às dinâmicas de interação social. Percebe-se que os dias atuais estão marcados pelo uso de instrumentos tecnológicos avançados que para além de serem técnicos, prolongam as capacidades do corpo humano e destinam-se a aumentá-las na relação do corpo com o mundo (SCHWONKE et al., 2011), destacando-se como tecnologias no âmbito da atenção à saúde os medicamentos, equipamentos, procedimentos técnicos, sistemas organizacionais, educacionais e de suporte, programas e protocolos assistenciais (LORENZETTI et al., 2012). Além disso, um dos avanços tecnológicos que vem tomando destaque no âmbito da saúde é o uso da informática mediante softwares.

As atividades de informática em saúde auxiliam os profissionais no processo de tomadas de decisões e prestação do cuidado em saúde (SANTOS, 2012), além de possuírem o papel de tornar as ações e situações da saúde populacional transparentes, a exemplo dos Sistemas de Informações em Saúde. Desse modo, devem estar sintonizadas com a legislação que rege o Sistema Único de Saúde.

Considerando que a informática apresenta-se como o instrumento a serviço da sociedade, o objetivo deste artigo foi realizar uma revisão integrativa sobre as publicações relativas ao desenvolvimento de softwares no Brasil na ótica das proposições do Decreto ${ }^{\circ} 7508$ de 28 de junho de 2011 para a organização e a integração das ações e dos serviços de saúde, segundo o qual deverá contemplar: a oferta de ações e serviços de vigilância em saúde, promoção, proteção e recuperação da saúde em âmbito regional e inter-regional (BRASIL, 2011).

\section{MÉTODOS}

Trata-se de uma revisão integrativa que segundo Mendes, Silveira e Galvão (2008), é um método que permite uma compreensão ampla do tema de interesse porque admite a inclusão simultânea de pesquisa experimental e quase-experimental e toma como base a Prática Baseada em Evidências, de modo que mediante a análise crítica de estudos relevantes, os seus resultados sejam utilizados na prática clínica. Ressalta-se que o método adotado para esta revisão segue as etapas recomendadas por estes autores, partindo do tema e da seguinte questão norteadora: As pesquisas brasileiras de desenvolvimento de softwares na área da saúde têm enfocado as proposições do Decreto $\mathrm{n}^{\circ} 7.508$ quanto à organização e a integração das ações e dos serviços de saúde? Na etapa seguinte foram definidos os modos de seleção dos materiais. 
Como critérios de inclusão foram selecionadas publicações entre 2008 e 2013, nos idiomas inglês, português e espanhol, tendo o Brasil como país de origem, relacionadas ao desenvolvimento, validação e/ou teste de softwares, focados na área da saúde, excluindo-se aquelas que abordassem softwares com finalidades educacionais para estudante e profissionais da área da saúde, uma vez que o foco do nosso estudo é a prática nos serviços (organização e integração das ações). As bases de dados para a busca de publicações foram: MEDLINE (Medical Literature Analysis and Retrieval System Online), LILACS (Literatura Latino-americana e do Caribe em Ciências da Saúde), SciELO (Scientific Electronic Library Online) e ACM Digital Library (Association for Computing Machinery Digital Library), com coleta de dados entre os dias 01 e 03 de dezembro de 2013.

As palavras-chave e operadores boleanos que serviram de busca foram: Software and (desenvolvimento or validação or teste) and saúde, ressaltando-se que sendo as bases LILACS e MEDLINE da área da saúde, esta última palavra não foi utilizada no campo de pesquisa.

$\mathrm{Na}$ terceira etapa foram definidas as informações a serem extraídas dos estudos selecionados, a saber: a instituição da qual faziam parte os autores; a finalidade principal; quem é o usuário e; a proposta do software. Seguiu-se uma quarta etapa de avaliação dos estudos e quais as suas conclusões. Ressalta-se aqui que a nossa intenção não é de analisar as adequações metodológicas dos estudos, interpretando resultados, e sim, na quinta etapa, apontar em qual direção o desenvolvimento de softwares está caminhando. Ainda assim, foi elaborada a tabela $1 \mathrm{em}$ que o leitor poderá ter um panorama geral das publicações, a partir de uma análise sintética dos apontamentos dos autores. Na sexta etapa apresenta-se a revisão/síntese do conhecimento, conforme resultados e discussão.

\section{RESULTADOS E DISCUSSÃO}

As palavras-chave remeteram a 155 resultados nas bases SciELO (68), ACM Library (47) e LILACS (41), com ausência na MEDLINE. Após a leitura cuidadosa dos resumos, considerando os critérios de inclusão e exclusão, foram selecionados cinco artigos de periódico científico no SciELO, cinco resultados na ACM Digital Library (4 artigos de anais de eventos científicos e 1 artigo de periódico científico) e nove artigos no LILACS, totalizando 10 publicações constituidoras da amostra final desta revisão, cujos dados principais estão disponíveis nas tabelas 1,2 e 3 . Caso o leitor deseje uma leitura detalhada do material selecionado, encontrará as citações nas referências ao fim do artigo.

\section{PERFIL DOS ESTUDOS SELECIONADOS}

Relativo aos anos de publicação, 52,9\% estava entre 2008 e 2010 e 47,1\% entre 2011 e 2013.

Na Figura 1 pode ser visto que a maioria dos estudos (12) pertencia a autores vinculados a instituições da região sudeste, sobretudo ao estado de São Paulo, seguida da região Nordeste (4), Sul (2) e Centro Oeste (1). Nenhum estudo estava vinculado à região Norte. Os valores ultrapassam 17, uma vez que em dois casos, para um mesmo estudo existiam autores de unidades federativas diferentes (um estudo vinculado ao mesmo tempo aos estados de São Paulo e Paraná e outro estudo ao de Pernambuco, São Paulo e Paraná). 
Quanto existia mais de um autor em um mesmo estudo para um mesmo estado, foi contabilizado apenas o valor um.

Apesar do pequeno quantitativo de estudos analisados e da não realização de cálculos per capita, os achados podem sinalizar a região sudeste, uma das mais favorecidas socioeconomicamente no Brasil, como quase hegemônica quanto se trata de publicações a respeito de softwares com foco na prática dos serviços e, por conseguinte, da construção dos mesmos. Já a região Norte, de menor poder aquisitivo, apresentou-se com ausência de autores no material selecionado, reproduzindo de certo modo, as desigualdades regionais presentes no Brasil. Apesar de a região Nordeste apresentar-se com o valor quatro, é preciso ressaltar que, de modo geral, são as capitais dessas regiões as que mais detêm o acesso a bens e serviços de saúde em detrimentos das cidades interioranas.

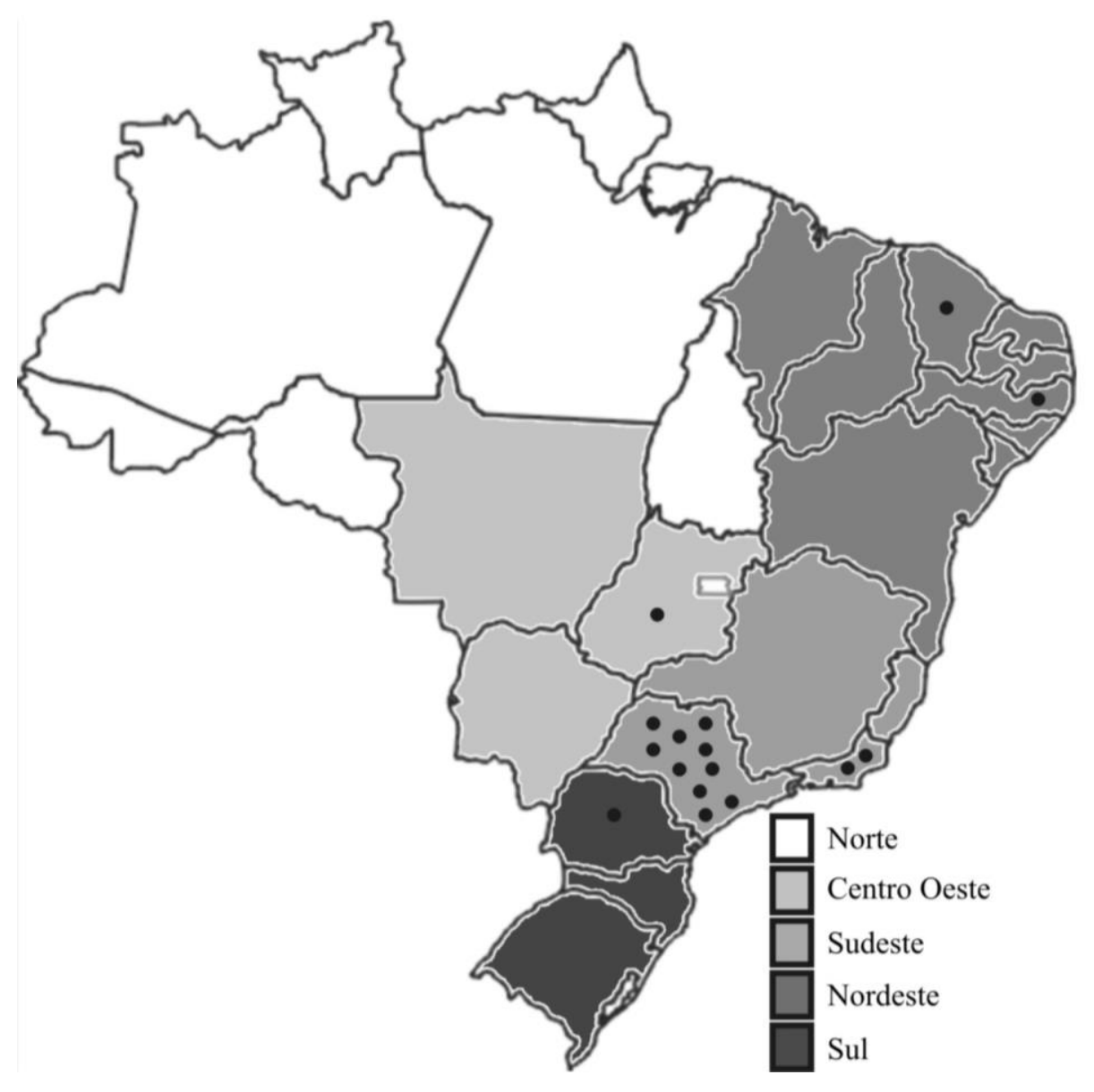

Figura 1: Distribuição dos estudos, segundo unidade da federação a que os autores estavam vinculados. Natal/RN, 2014.

\section{RELAÇÃO COM A ORGANIZAÇÃO E A INTEGRAÇÃO DAS AÇÕES E DOS SERVIÇOS}

No que concerne à finalidade principal dos softwares nos estudos selecionados (tabela 2), observa-se que a maioria destinava-se a organização dos serviços (47\%, três dos quais relacionados à melhoria da comunicação entre profissionais e profissionais-usuários, quatro ao armazenamento de dados de pacientes e um ao dimensionamento de profissionais, vide tabela 1 para detalhamento das propostas dos softwares). Em seguida 
houve destaque para a finalidade de diagnóstico $(29,4 \%$, quatro dos quais a respeito de imagens de diagnóstico e um da análise da situação de saúde de trabalhadores mediante o seu monitoramento). Contudo, houve pequeno percentual $(23,6 \%$ na soma geral) no tocante: ao monitoramento (Software de preenchimento autoadministrado sobre alimentação por escolares; Sistema de vigilância da população de Aedes Aegypiti), à prevenção de doenças (Jogo de entretenimento a respeito de educação em saúde, acoplado a uma esteira ergométrica), e ao tratamento (Jogo com sessões de terapia familiar, mediante perguntas e repostas).

Esclarece-se que a finalidade principal aqui citada diz respeito à finalidade última do software explicitada pelos autores. Desse modo, um software com finalidade principal de monitoramento, por exemplo, pode também está relacionado à organização.

TABELA 1: Material selecionado nas bases de dados consultadas. Natal/RN, 2014.

Continua...

\begin{tabular}{|c|c|c|c|c|}
\hline $\mathrm{N}$ & TÍTULO & AUTOR (ES) & ANO & PROPOSTA \\
\hline 1 & $\begin{array}{l}\text { Protocolo eletrônico de coleta de } \\
\text { dados clínicos da microcirurgia } \\
\text { endoscópica transanal (TEM): } \\
\text { desenvolvimento e aplicação }\end{array}$ & $\begin{array}{l}\text { OLIVEIRA, M. M. } \\
\text { et al. }\end{array}$ & 2009 & Criar base de dados clínicos. \\
\hline 2 & $\begin{array}{l}\text { Regulação médica em emergência } \\
\text { pela plataforma web: um estudo } \\
\text { piloto. }\end{array}$ & $\begin{array}{l}\text { ADOLFI JÚNIOR, } \\
\text { M. S. et al. }\end{array}$ & 2010 & $\begin{array}{c}\text { Agilizar os encaminhamentos de } \\
\text { pacientes, segundo gravidade de } \\
\text { caso. }\end{array}$ \\
\hline 3 & $\begin{array}{l}\text { Avaliação de um Serviço de } \\
\text { Gerenciamento de Sessão para } \\
\text { Ambientes de Medicina Ubíqua. }\end{array}$ & $\begin{array}{l}\text { DINIZ, J. R. B. et } \\
\text { al. }\end{array}$ & 2008 & $\begin{array}{l}\text { Comunicação entre médicos e } \\
\text { disponibilidade de prontuários } \\
\text { em qualquer hora e lugar. }\end{array}$ \\
\hline 4 & $\begin{array}{l}\text { Using Machine Learning Classifiers } \\
\text { to Assist Healthcare-Related } \\
\text { Decisions: Classification of } \\
\text { Electronic Patient Records }\end{array}$ & $\begin{array}{l}\text { POLLETTINI, J.T. } \\
\text { et al. }\end{array}$ & 2012 & $\begin{array}{l}\text { Acessar o histórico médico do } \\
\text { paciente e inserir novas } \\
\text { informações, recomendando-se } \\
\text { níveis de vigilância na prestação } \\
\text { do cuidado. }\end{array}$ \\
\hline 5 & $\begin{array}{l}\text { Dimensionamento informatizado de } \\
\text { profissionais de enfermagem: } \\
\text { avaliação de um software. }\end{array}$ & $\begin{array}{l}\text { PEREIRA, I. M. et } \\
\text { al. }\end{array}$ & 2011 & $\begin{array}{c}\text { Promover o dimensionamento de } \\
\text { profissionais de instituições } \\
\text { hospitalares. }\end{array}$ \\
\hline 6 & $\begin{array}{c}\text { Avaliação de relatório eletrônico de } \\
\text { anestesia. }\end{array}$ & $\begin{array}{l}\text { ZAMPER, R. P. C. } \\
\text { et al. }\end{array}$ & 2010 & $\begin{array}{l}\text { Recuperar automaticamente os } \\
\text { dados de um sistema de } \\
\text { informações hospitalares pré- } \\
\text { existente (identificação, } \\
\text { avaliação } \\
\text { pré-operatória e resultados de } \\
\text { exames laboratoriais). }\end{array}$ \\
\hline 7 & $\begin{array}{l}\text { Validação do prontuário eletrônico do } \\
\text { paciente em uma instituição de ensino } \\
\text { superior em saúde: relato da } \\
\text { experiência no módulo Anamnese. }\end{array}$ & $\begin{array}{l}\text { BRAGA, R. D. et } \\
\text { al. }\end{array}$ & 2013 & $\begin{array}{c}\text { Melhorar a comunicação entre os } \\
\text { profissionais em ambientes } \\
\text { hospitalares. }\end{array}$ \\
\hline 8 & $\begin{array}{l}\text { O papel da interação centrada ao } \\
\text { usuário para suporte à colaboração em } \\
\text { uma unidade de emergência médica. }\end{array}$ & $\begin{array}{l}\text { ANDRADE, R.; } \\
\text { VIVACQUA, A. S.; } \\
\text { GARCIA, A. C. B. }\end{array}$ & 2012 & $\begin{array}{c}\text { Realizar o registro dos } \\
\text { prontuários em um sistema } \\
\text { eletrônico }\end{array}$ \\
\hline 9 & $\begin{array}{l}\text { Desenvolvimento e avaliação da } \\
\text { usabilidade de um recordatório de } 24 \\
\text { horas estruturado e computadorizado } \\
\text { para acompanhamento do consumo } \\
\text { alimentar de escolares. }\end{array}$ & $\begin{array}{l}\text { RUGGERI, B. F. } \\
\text { F. }\end{array}$ & 2011 & $\begin{array}{l}\text { Permitir o preenchimento } \\
\text { autoadministrado do } \\
\text { Recordatório por escolares. }\end{array}$ \\
\hline
\end{tabular}


TABELA 1: Material selecionado nas bases de dados consultadas. Natal/RN, 2014. Conclusão.

\begin{tabular}{|c|c|c|c|c|}
\hline $\mathrm{N}$ & TÍTULO & AUTOR (ES) & $\mathrm{ANO}$ & PROPOSTA \\
\hline 10 & $\begin{array}{c}\text { An entomological surveillance system } \\
\text { based on open spatial information for } \\
\text { participative dengue control }\end{array}$ & REGIS, L. et al. & 2009 & $\begin{array}{c}\text { Estimar as populações de } \\
\text { mosquitos a partir da contagem } \\
\text { de ovos de ovitrampas. }\end{array}$ \\
\hline 11 & $\begin{array}{l}\text { iFitPro: tecnologia interativa para a } \\
\text { prática de atividades físicas }\end{array}$ & SILVA, A. G. et al. & 2008 & $\begin{array}{l}\text { Praticante de atividade física } \\
\text { interagir com um jogo sobre } \\
\text { alimentação em uma máquina } \\
\text { ergométrica. }\end{array}$ \\
\hline 12 & $\begin{array}{l}\text { A inovação tecnológica como } \\
\text { ferramenta para monitoramento da } \\
\text { saúde dos trabalhadores de } \\
\text { enfermagem }\end{array}$ & $\begin{array}{l}\text { BAPTISTA, P. C. } \\
\text { P. et al. }\end{array}$ & 2011 & $\begin{array}{l}\text { Captar os agravos a partir da } \\
\text { notificação do sistema. }\end{array}$ \\
\hline 13 & $\begin{array}{c}\text { Processamento de estruturas } \\
\text { tridimensionais de Medicina Nuclear } \\
\text { na modalidade PET }\end{array}$ & $\begin{array}{c}\text { FLÓREZ } \\
\text { PACHECO, E.; } \\
\text { FURUIE, S. S. }\end{array}$ & 2013 & $\begin{array}{c}\text { Diminuir ruídos em imagens, } \\
\text { por meio de filtros e ferramenta } \\
\text { de segmentação. }\end{array}$ \\
\hline 14 & $\begin{array}{l}\text { Avaliação computacional de enfisema } \\
\text { pulmonar em TC: comparação entre } \\
\text { um sistema desenvolvido localmente e } \\
\text { um sistema de uso livre. }\end{array}$ & $\begin{array}{l}\text { FELIX, J. H. S. et } \\
\text { al. }\end{array}$ & 2009 & $\begin{array}{l}\text { Reunir ferramentas de } \\
\text { segmentação do parênquima } \\
\text { pulmonar. }\end{array}$ \\
\hline 15 & $\begin{array}{l}\text { Software livre e de código aberto para } \\
\text { avaliação de imagens de } \\
\text { angiotomografia de coronárias. }\end{array}$ & $\begin{array}{l}\text { HADLICH, M. S. } \\
\text { et al. }\end{array}$ & 2012 & $\begin{array}{l}\text { Viabilizar software de domínio } \\
\text { público para avaliação de } \\
\text { exames de angiotomografia. }\end{array}$ \\
\hline 16 & $\begin{array}{l}\text { Postural assessment software } \\
\text { (PAS/SAPO): validation and } \\
\text { reliabiliy. }\end{array}$ & $\begin{array}{l}\text { FERREIRA, E. A. } \\
\text { G. et al. }\end{array}$ & 2010 & $\begin{array}{c}\text { Auxiliar a avaliação da postura } \\
\text { a partir de fotos digitalizadas. }\end{array}$ \\
\hline 17 & $\begin{array}{l}\text { Uso de Senso Comum pelo Terapeuta } \\
\text { em Jogos Computacionais para } \\
\text { Promover a Aproximação da Família }\end{array}$ & $\begin{array}{c}\text { ROSAS } \\
\text { VILLENA, J. M.; } \\
\text { ANACLETO, J. C. }\end{array}$ & 2009 & $\begin{array}{c}\text { Auxiliar sessões de terapia com } \\
\text { uso do senso comum }\end{array}$ \\
\hline
\end{tabular}

TABELA 2: Classificação dos achados segundo finalidade principal do software.

Natal/RN, 2014.

\begin{tabular}{lccc}
\hline FINALIDADE & N & $\%$ & $\begin{array}{l}\text { RELAÇÃO DA SAÚDE COM O DECRETO No 7.508 } \\
\text { 2011 (Vigilância, Promoção, Proteção e Recuperação) }\end{array}$ \\
\hline Organização dos serviços & 8 & 47,0 & Vigilância (controle) \\
Diagnóstico & 5 & 29,4 & Vigilância (controle) \\
Monitoramento & 2 & 11,8 & Proteção / Vigilância (prevenção) \\
Prevenção de doenças & 1 & 5,9 & Vigilância (controle) \\
Tratamento & 1 & 5,9 & Recuperação / Vigilância (proteção) \\
\hline TOTAL & 17 & \\
\hline
\end{tabular}

Em relação ao número maior de proposições de softwares relacionados à organização dos serviços, sobretudo quanto ao armazenamento de dados de usuários, salienta-se a importância da informação em saúde no o reconhecimento da situação de grupos sociais específicos (DUARTE; TEDESCO; PARCIANELLO, 2012) para que sejam analisadas, gerenciadas e tomadas decisões mais coerentes com as necessidades e capacidades dos prestadores de cuidado (SANTOS et al., 2012) e com a tecnologia proporcionado a agilidade necessária aos sistemas de saúde (OLIVEIRA, 2012).

Observa-se que tais softwares destinados à organização restringiam-se aos locais de trabalho dos profissionais de saúde. Assim, na ausência de determinado profissional, o acesso às informações dos usuários do serviço torna-se limitada, quando a tecnologia atual pode favorecer a comunicação entre pessoas mesmo à distância. A literatura aponta que 
uma das desvantagens no uso de softwares é a ineficiência na troca de dados em qualquer hora e em qualquer lugar (TORRES; CAMPOS, 2014). Nesta revisão, apenas um estudo apresentava esta proposta.

A presença de software para fins de diagnóstico também foi vista positivamente, considerando que este quando não realizado corretamente, culmina em risco a vida, além de desperdícios de recursos nos serviços. A maioria era referente ao uso de software para diagnóstico por imagem de boa qualidade, campo que tem avançado no Brasil e que permite precisão em procedimento e qualidade em exames por imagens (SALES; OLIVEIRA; SPIRANDELLI et al., 2012). Salienta-se, contudo que imagens por si só correspondem a pouco, requerendo profissionais capacitados para a sua interpretação, além de avaliação clínico-ambulatorial com escuta qualificada do usuário para elevar a precisão do diagnóstico.

Foi visto de modo negativo um menor quantitativo de estudos de monitoramento, prevenção e tratamento de doenças, além da ausência de qualquer um relativo à promoção da saúde. Além disso, em nossa revisão houve apenas um estudo abordando a educação em saúde (estudo 11, prevenção, tabela 1). Tal escassez corrobora a revisão de Santos et al. (2012) para a área de fonoaudiologia em que houve ausência de estudos que utilizassem softwares nas áreas educacional e de saúde coletiva. Os estudos 10 e 11 a prevenção de doenças, sendo o de $\mathrm{n}^{\circ} 10$ voltado para a vigilância em saúde, inovando por criar um software brasileiro de acesso livre para dados geoespaciais. De fato, Cavicchioli Neto et al. (2014), ao discutirem sobre o uso de ferramentas computacionais em geoprocessamento escrevem sobre a dificuldade de encontrar aquelas que levem em consideração as dinâmicas sociais e os ecossistemas em doenças específicas, uma vez que os softwares envolvem patentes e pessoal especializado.

Correlacionando-se a finalidade dos estudos com o decreto $\mathrm{n}^{\circ} 7.508 / 2011$, positivamente, todos apresentavam relação com a vigilância em saúde (tabela 1), caracterizada pelo o artigo $2^{\circ}$ da Portaria $n^{\circ} 1.378$, de 09 de julho de 2013 (BRASIL, 2013, p.48) como um "processo contínuo e sistemático de coleta, consolidação, análise e disseminação de dados sobre eventos relacionados à saúde (...) para a proteção da saúde da população, a prevenção e controle de riscos, agravos e doenças, bem como para a promoção da saúde". Proteção aqui entendida como controlar possíveis riscos à saúde presentes no modo como a sociedade se organiza (BARBOSA; COSTA, 2010). Portanto, este conceito de vigilância engloba claramente a organização e a integração das ações e dos serviços de saúde presentes no Decreto, isto é, os softwares nos materiais analisados abordando a vigilância poderiam englobar também a promoção e proteção da saúde, além da recuperação, encontrada em um estudo.

Porém, apesar dos estudos contemplarem a vigilância em saúde, houve ausência de softwares de promoção da saúde, o que pode ser compreendido quanto analisamos a quem os mesmos se destinam. Quanto aos usuários finais dos softwares (tabela 3), sete estudos destinavam-se aos profissionais da saúde, dois a populações-chave (escolares e praticantes de atividade física), um ao público em geral, um a profissionais e graduandos e outro a profissionais e usuários do serviço. Cinco estudos não explicitavam claramente nos estudos quem seriam os usuários, contudo na leitura dos mesmos fica compreendido que o uso era para profissionais. Portanto, percebe-se a "massividade" dos estudos quanto a destinar softwares aos profissionais (12 - 70,6\%), em uma quase supressão da população em geral. 
Tal achado é atribuído ao fato da escassez nos trabalhos analisados de softwares nos quais os usuários dos serviços de saúde possam participar como atores principais (prevenção e tratamento), com ausência daqueles destinados à promoção da saúde. Softwares que trabalham nesta perspectiva se encaminham para o cuidado centrado no usuário, modelo que segundo Martins et al. (2011) requer a adoção de meios para que o cidadão possa se expressar e transformar a realidade por ele vivida, onde ambos, sociedade e Estado apresentam deveres e direitos, sendo a falta de participação da população atribuída não somente à falta de infraestrutura e recursos, mas também à uma falta de cultura cívica de participação e ausência de interesse político para tal.

TABELA 3: Classificação dos estudos segundo usuário final do software. Natal/RN, 2014.

\begin{tabular}{lcc}
\hline USUÁRIO & N & $\%$ \\
\hline Profissionais da saúde & 7 & 41,2 \\
Sem identificação explícita do usuário & 5 & 29,4 \\
Populações-chave & 2 & 11,8 \\
Profissionais e graduandos & 1 & 5,9 \\
Profissionais e usuários dos serviços & 1 & 5,9 \\
Qualquer pessoa & 1 & 5,9 \\
\hline TOTAL & \multicolumn{2}{c}{17} \\
\hline
\end{tabular}

Ressalta-se o ponto de vista de Honorato (2014) de que as áreas transdisciplinares costumam entender o universo virtual como meio de acesso ou contato com os sujeitos e não como campo de interação com estes, quando a integração entre saúde pública com a cibercultura deveria ser uma via de aplicação de interagir apara promover políticas públicas, intervenções e pesquisas em saúde pública.

\section{CONCLUSÕES}

Nos estudos analisados foi visto um direcionamento de publicações voltadas à construção, validação ou teste de softwares para a região Sudeste, sobretudo para o estado de São Paulo. Tal achado pode indicar concentração do uso de tecnologias computacionais para tal região quando o ideal seria a disponibilidade das mesmas em todas as regiões do Brasil.

Nas publicações analisadas, a maior parte dos softwares se encaminhava para conduzir a organização dos serviços e facilitar ou aperfeiçoar o diagnóstico de doenças. Considerando o potencial dos recursos computacionais quanto à possibilidade da projeção de softwares destinados a variados objetivos, esta revisão encontrou carência de estudos quando se tratava do monitoramento da situação de saúde/doença, ao tratamento e a prevenção de doenças.

A ausência de softwares para a promoção da saúde aqui encontrada segue um antigo "gargalo" nos serviços de saúde, onde as ações de promoção que poderiam evitar ou diminuir as estatísticas de acometimento de doenças são pouco vislumbradas no meio acadêmico, culminando em uma valorização de estudos voltados para a doença os quais possuem grande valor, mas que necessitam estarem atrelados a práticas que evitem a superlotação dos serviços por causas preveníveis a exemplo de boa parte das doenças crônico-degenerativas que afligem a população mundial contemporânea. 
Os estudos relacionam-se às especificações analisadas do Decreto $\mathrm{n}^{\circ} 7508 / 2011$, sobretudo no que tange à vigilância em saúde, demonstrando sintonia com a legislação vigente no Brasil, com foco nos profissionais como usuários finais. A escassez encontrada de foco na população geral como usuária distancia-se da perspectiva da coparticipação desta no cuidado e nas ações voltadas para a sua saúde, em uma posição de passividade no uso de softwares.

Conclui-se que as proposições dos softwares podem trazer benefícios para a organização e integração das ações e dos serviços de saúde. Contudo, considerando as variadas possibilidades de uso que a tecnologia pode proporcionar, os estudos analisados apontam a necessidade de ampliar o leque de opções quanto a finalidade e aos usuários.

Apesar do pequeno número de estudos analisados, esta revisão pode contribuir para que pesquisadores da área cogitem novas vias de destino para os softwares na área da saúde.

\section{REFERÊNCIAS BIBLIOGRÁFICAS}

1. CAVICCHIOLI NETO, V. et al. Desenvolvimento e Integração de Mapas Dinâmicos Georreferenciados para o Gerenciamento e Vigilância em Saúde. J. Health Inform., São Paulo, v. 6, n. 1, p. 3-9, 2014.

2. DINIZ, J. R. B. et al. Avaliação de um Serviço de Gerenciamento de Sessão para Ambientes de Medicina Ubíqua. In: XIV SIMPÓSIO BRASILEIRO DE SISTEMAS MULTIMÍDIA E WEB, 2008, Vila Velha. Anais... Nova Iorque: ACM New York, 2008, p.4-11.

3. DUARTE, M. L. C.; TEDESCO, J. R.; PARCIANELlO, R. R. O uso do sistema de informação na estratégia saúde da família: percepções dos enfermeiros. Rev Gaúcha Enferm., Porto Alegre, v. 33, n. 4, p.111-117, 2012.

4. FELIX, J. H. S. et al . Avaliação computacional de enfisema pulmonar em TC: comparação entre um sistema desenvolvido localmente e um sistema de uso livre. J. bras. pneumol., São Paulo, v. 35, n. 9, p. 868-876, 2009.

5. FERREIRA, E. A. G. et al . Postural assessment software (PAS/SAPO): validation and reliabiliy. Clinics, São Paulo, v. 65, n. 7, p. 675-681, 2010.

6. FLOREZ PACHECO, E.; FURUIE, S. S. Processamento de estruturas tridimensionais de Medicina Nuclear na modalidade PET. Rev. Bras. Eng. Bioméd., Rio de Janeiro, v. 29, n. 1, p. 70-85, 2013.

7. HADLICH, M.S.et al . Software livre e de código aberto para avaliação de imagens de angiotomografia de coronárias. Arq. Bras. Cardiol., São Paulo, v. 99, n. 4, p. 944-951, 2012 .

8. HONORATO, E. J. S.A. A interface entre Saúde Pública e Cibercultura. Ciênc. saúde coletiva, Rio de Janeiro, v. 19, n.2, p. 481-485, 2014.

9. LORENZETTI, J. et al . Tecnologia, inovação tecnológica e saúde: uma reflexão necessária. Texto contexto - enferm., Florianópolis, v. 21, n. 2, p.432-439, 2012.

10. MARTINS, P. C. et al. De quem é o SUS? Sobre as representações sociais dos usuários do Programa Saúde da Família. Ciênc. saúde coletiva, Rio de Janeiro, v. 16, n. 3, p. 1933-1942, 2011. 
11. MENDES, K. D.S.; SILVEIRA, R. C. C. P.; GALVAO, C. M. Revisão integrativa: método de pesquisa para a incorporação de evidências na saúde e na enfermagem. Texto contexto - enferm., Florianópolis, v. 17, n. 4, p. 758-64, 2008.

12. OLIVEIRA, J. F. Gestão de Tecnologias da Informação e da Comunicação na Saúde: uma análise sobre o uso do prontuário eletrônico. Interface, Natal, v.9, n.1, p. 7-25, 2012.

13. OLIVEIRA, M.M. et al. Protocolo eletrônico de coleta de dados clínicos da microcirurgia endoscópica transanal (TEM): desenvolvimento e aplicação. $A B C D$, arq. bras. cir. dig., São Paulo, v.22, n.4, p. 216-221, 2009.

14. PEREIRA, I. M. et al . Dimensionamento informatizado de profissionais de enfermagem: avaliação de um software. Rev. esc. enferm. USP, São Paulo, v. 45, n. Esp, p. 1600-1605, 2011 .

15. POLLETTINI, J.T. et al. Using Machine Learning Classifiers to Assist HealthcareRelated Decisions: Classification of Electronic Patient Records. Journal of Medical Systems, Nova Iorque, v. 36, p. 9859, 2012.

16. REGIS, L. et al. An entomological surveillance system based on open spatial information for participative dengue control. An. Acad. Bras. Ciênc., Rio de Janeiro, v. 81, n. 4, 655-662, 2009.

17. ROSAS VILlENA, J. M.; ANACLETO, J. C. Uso de Senso Comum pelo Terapeuta em Jogos Computacionais para Promover a Aproximação da Família. In: SIMPÓSIO BRASILEIRO DE SISTEMAS MULTIMÍDIA E WEB (WEBMEDIA), 2009, Fortaleza. Anais... Nova Iorque: ACM, New York, 2009, artigo 38.

18. RUGGERI, B. F. F. Desenvolvimento e avaliação da usabilidade de um recordatório de 24 horas estruturado e computadorizado para acompanhamento do consumo alimentar de escolares. 2011. 133 p. Dissertação (Mestrado em Nutrição e Saúde Pública) - Faculdade de Saúde Pública, Universidade de São Paulo, São Paulo, 2011.

19. SAlES, O. P.; OLIVEIRA, C. C. C.; SPIRANDELli; M. F. A.P. Atuação de enfermeiros em um Centro de Diagnóstico por Imagem. J. Health Sci. Inst., São Paulo, v. 28, n.4, p.325-328, 2010.

20. SANTOS, K.W. Utilização de softwares em pesquisas científicas de fonoaudiologia. $J$. Health Inform., São Paulo, v.4, n.2, p.55-8, 2012.

21. SCHWONKE, C. R. G. B. et al. Perspectivas filosóficas do uso da tecnologia no cuidado de enfermagem em terapia intensiva. Rev. bras. enferm., Brasília, v. 64, n. 1, p. 189-192, 2011.

22. SILVA, A. G. et al. C. iFitPro: tecnologia interativa para a prática de atividades físicas. In: IHC 2008 - VIII SIMPÓSIO BRASILEIRO SOBRE FATORES HUMANOS EM SISTEMAS COMPUTACIONAIS, 2008, Porto Alegre. Anais... Porto Alegre: Sociedade Brasileira de Computação, 2008, p. 324-325.

23. TORRES, A. A. L.; CAMPOS, V. Evernote como ferramenta de organização de informações em saúde. Revista Eletrônica Gestão \& Saúde, Brasília, v. 5, n. 2, p.50113, 2014.

24.ZAMPER, R. P. C. et al. Avaliação de relatório eletrônico de anestesia. Rev. Bras. Anestesiol., Campinas, v. 60, n. 3, p. 285-301, 2010. 